\title{
LIBERTAD DE EXPRESIÓN: APUNTES SOBRE SU RECONOCIMIENTO Y CONTENIDO EN EL TRABAJO
}

\author{
FREEDOM OF EXPRESSION: NOTES ON ITS RECOGNITION \\ AND CONTENT AT WORK
}

JOSÉ LUIS UGARTE CATALDO*

\section{Resumen}

Este trabajo analiza la libertad de expresión en el ámbito de las relaciones de trabajo y sus límites con énfasis en el derecho chileno.

\section{Palabras Clave}

Libertad de expresión, contrato de trabajo, derecho chileno.

\begin{abstract}
This paper analyzes freedom of expression in the field of employment relationships and its limits with emphasis on Chilean law.
\end{abstract}

\section{Keywords}

Freedom of expression, contract of employment, chilean law.

\section{LIBERTAD DE EXPRESIÓN EN EL TRABAJO: RECONOCIMIENTO Y CONTENIDO}

Pocas dudas caben que la relevancia de la "voz" en el trabajo se vincula a la autonomía. Como expresión de la riqueza individual de la persona que 
participa del proceso productivo, pero también como la manifestación de la acción colectiva de los trabajadores.

Pero esa expresión tan singular de la autonomía humana como es la "voz", se enfrenta en el trabajo con un escenario cargado de complejidad. Como sugiere Budd la expresión del trabajador queda peligrosamente sometida a la racionalidad empresarial: "la voz se identifica con la implicación del trabajador a la hora de compartir ideas para mejorar los procesos (ej. círculos de calidad) o con el hecho de que el trabajador se desarrolle en grupos con autonomía (ej. equipos de trabajo que se dirigen a si mismo), todo ello a fin de mejorar el rendimiento de la organización". De ahí que "ver el trabajo como ciudadanía profesional, sin embargo, es ir más allá de los aspectos de eficiencia de la voz, para dar valor también a las necesidades humanas, incluso cuando la voz del trabajador no mejora la productividad"1.

La libertad de expresión es un derecho ampliamente reconocido en la normativa del derecho internacional e interna. El artículo 1, número 1, de la Convención Americana de Derechos Humanos señala que "toda persona tiene derecho a la libertad de pensamiento y de expresión. Este derecho comprende la libertad de buscar, recibir y difundir informaciones e ideas de toda índole, sin consideración de fronteras, ya sea oralmente, por escrito o en forma impresa o artística o por cualquier otro procedimiento de su elección".

A su turno el artículo 19, numero 12 de la Constitución chilena asegura a todas las personas: "La libertad de emitir opinión y la de informar, sin censura previa, en cualquier forma y por cualquier medio, sin perjuicio de responder de los delitos y abusos que se cometan en el "ejercicio de estas libertades, en conformidad a la ley, la que deberá ser de quórum calificado".

Más allá de la configuración jurídica de este derecho ${ }^{2}$, cuestión directamente vinculada a cada tradición legal, la libertad de expresión

Artículo recibido para su evaluación el 10 de mayo de 2020, y aprobado para su publicación el 24 de julio de 2020.

* Doctor en Derecho, Profesor e Investigador de la Universidad Diego Portales. jose.ugarte@udp.cl

1 BUDD, J.: El pensamiento sobre el trabajo. Tirant lo Blanch, Valencia, 2015. p 138.

2 Se puede mirar su estructura como un solo derecho con un contenido complejo y binario (expresión+ información), o como dos derechos distintos pero relacionados, cuestión que dependerá de la formula normativa en que cada tradición legal concrete estos derechos fundamentales. Ahora, sea como sea, la libertad de expresión opera como condición necesaria de la información. 
comprende un conjunto de posiciones activas protegidas en favor de su titular, en este caso, del trabajador:

Por una parte, la facultad para difundir ideas y discursos de cualquier naturaleza o índole y por cualquier medio, en cualquier momento y oportunidad, sin autorización previa (libertad de expresión). Y por otra, la facultad emitir información y/o recibir información, esto es, "la difusión de hechos, sucesos o acontecimientos de la realidad. En ese sentido, comprende el derecho a difundir libremente información. Pero no solo es el derecho a difundir o transmitir la información lo que se tutela, sino también el derecho de acceder a ella"3.

La dogmática constitucional ha destacado la amplitud del contenido de este derecho fundamental sosteniendo que consiste en la facultad de toda persona de exteriorizar, por el medio que crea conveniente, sus ideas, creencias y pensamientos. Esto es, "la libertad de expresión comprende la libertad de buscar, recibir y difundir informaciones e ideas de toda índole, sin consideración de fronteras, ya sea oralmente, por escrito o en forma impresa o artística, o por cualquier otro procedimiento de la elección del que la ejercita, conllevando este derecho consigo el derecho a permanecer en silencio cuando se requiere a una persona que exprese una idea o una opinión que no comparte"4.

Se da cobertura constitucional, entonces, a todo tipo de discurso o expresión, particularmente el crítico. En efecto, como ha señalado la jurisprudencia comparada la libertad de expresión "tiene por objeto el derecho a la libertad de expresión tiene por objeto la libre expresión de pensamientos, ideas y opiniones, concepto amplío dentro del cual deben incluirse las creencias y juicios de valor. Este derecho comprende la crítica de la conducta de otro, aun cuando la misma sea desabrida y pueda molestar, inquietar o disgustar a quien se dirige, pues así lo requieren el pluralismo, la tolerancia y el espíritu de apertura, sin los cuales no existe sociedad democrática" (Tribunal Constitucional Español, STC 6/2000). En el mismo sentido, la jurisprudencia constitucional alemana ha señalado que "la protección se refiere no sólo al contenido de la manifestación, sino también a su forma. El que una afirmación se formule de forma polémica

3 MARCIANI, B.: El derecho a la libertad de expresión y la tesis de los derechos preferentes. Palestra, Lima, 2004. p 115.

4 MEDINA, C.: "La libertad de expresión". En: AAVV Sistema Jurídico y Derechos Humanos, El derecho nacional y las obligaciones internacionales de Chile en materia de Derechos Humanos. Universidad Diego Portales, Santiago de Chile, 1996. p 149. 
o hiriente no la sustrae del ámbito de protección del derecho fundamental" (Tribunal Constitucional alemán, BVerfGE 93, 266, 10.10.1995).

Así también, la jurisprudencia internacional ha señalado que la libertad de expresión "no sólo se protege la emisión de expresiones inofensivas o bien recibidas por la opinión pública, sino también la de aquellas que chocan, irritan o inquietan a los funcionarios públicos o a un sector cualquiera de la población" (Corte Interamericana de Derechos Humanos, Caso Kimel vs Argentina, 2008, párrafo 88).

La protección extensa en términos de continente y contenido forma y fondo es relevante para un contexto como la empresa: según ya hemos explicado la situación de sujeción del trabajador como resultado de someterse a un poder organizacional ajeno -la denominada subordinación laboral-, hace especialmente relevante la tutela del discurso crítico y conflictivo, como forma de superar el inhóspito escenario que para ese derecho configura generalmente el espacio empresarial.

Desde la perspectiva jurídica, cabe destacar, la amplitud de los medios a través de los cuales se ejerce este derecho. Como se ha explicado "las expresiones se pueden realizar a través de palabras y letras; pero también por medio de actos (como la quema de bandera) y símbolos (como lo sería llevar un brazalete con algún mensaje). Se ha protegido el valor comunicativo del silencio (por ejemplo, en casos de persona que se han negado a prestar juramentos) y las artes, como las pinturas, la música y las obras de teatro, han sido consideradas, también, formas de expresión. Las protestas, en fin, son también formas de expresión"5.

Particularmente relevante en materia laboral es, precisamente, la libertad de expresión que se ejerce por la vía de la protesta. Y si bien se trata de un derecho que podrá ser ejercido de manera individual por el trabajador en el ámbito del trabajo -por ejemplo, por condiciones de trabajo o por incumplimientos laboral de la empresa-, su máxima expresión es su ejercicio colectivo como cobertura jurídica de las acciones complementarias del derecho de huelga, legitimando las diversas manifestaciones que permiten hacer visible el conflicto laboral, tales como las manifestaciones, piquetes y marchas entre otras.

5 LOVERA, D.: "Libertad de expresión, derecho de reunión y protesta en la Constitución". En: AAVV La Constitución chilena. Bassa et alt (editores). LOM editores, Santiago, 2015. p 103. 
Desde el punto de vista pasivo, esto es, desde la perspectiva de los sujetos afectos al contenido del derecho de libertad de expresión del trabajador, nos referiremos al caso del empleador ${ }^{6}$.

Respecto del empleador -público o privado- la libertad de expresión del trabajador supone un conjunto de prohibiciones:

En primer lugar, la prohibición de censura y de cualquier restricción previa del ejercicio del derecho de libertad de expresión y/o información. Entendiendo por censura previa como "cualquier impedimento o medida ilegitimo, de carácter preventivo que limite o dificulte el ejercicio de la libertad de expresión"?.

De este modo, cualquier conducta del empleador que restrinja o impida el ejercicio de la libertad de expresión y de información por parte del trabajador, supone una lesión ese derecho fundamental.

En segundo lugar, la prohibición de represalias por el ejercicio del derecho a libertad de expresión en el sentido amplio ya explicado. Por represalia debe entenderse cualquier conducta del empleador que afecte al trabajador como consecuencia del ejercicio de ese derecho fundamental.

En este caso, dicha represalia puede ser desde una conducta material -un perjuicio económico o un llamado de atención- hasta conductas jurídicas -como una amonestación reglamentaria- y particularmente el despido. Mirado desde este punto de vista, podríamos hablar de una garantía de indemnidad propia de la libertad de expresión que a diferencia de la garantía común protege las represalias por el ejercicio de la libertad de expresión, sin requerir el denominado elemento institucional, esto es la intervención de la Inspección del Trabajo o los Tribunales de Justicia.

La garantía de no ser objeto de represalias por el ejercicio de la libertad de expresión ha tenido reconocimiento tanto en la jurisprudencia

6 Es obvio que respecto del Estado también pesan deberes en relación a la libertad de expresión del trabajador, tanto negativos -como la prohibición de censura-, como deberes positivos. En este último caso, particularmente relevantes es que el ejercicio real y efectivo de la libertad de expresión "no depende simplemente del deber del Estado de abstenerse de cualquier injerencia, sino que puede requerir medidas positivas de protección incluso en las relaciones entre las personas. En efecto, en ciertos casos, el Estado tiene la obligación positiva de proteger el derecho a la libertad de expresión, incluso frente a ataques provenientes de particulares” (Corte Interamericana de Derechos Humanos, caso Lagos del Campo vs, Perú, sentencia del 31 de agosto del 2017, párrafo 93).

7 GONZÁLEZ, F.: "Censura judicial y libertad de expresión: Sistema interamericano y derecho chileno". En: AAVV. Libertad de Expresión en Chile. Universidad Diego Portales, Santiago, 2006. p 13. 
internacional, como en la nacional. En el primer caso la Corte Interamericana ha sostenido que "la libertad de expresión requiere que nadie sea arbitrariamente menoscabado" por manifestar su propio pensamiento" (Corte Interamericana de Derechos Humanos, caso Lagos del Campo vs, Perú, sentencia del 31 de agosto del 2017, párrafo 89). En el segundo caso, los tribunales chilenos han reconocido como despidos lesivos de derechos fundamentales del trabajador los que correspondieron a represalias por declaraciones efectuadas por el trabajador: "cabe señalar que la medida en discordia (despido) es una consecuencia ex post, es decir un efecto que se despliega solo después de la difusión de la opinión en redes sociales, por lo tanto, en rigor no existe censura sino eventualmente represalia. Luego, una represalia, bajo una concepción amplia, también importa un impedimento dificultad o escollo a la libre expresión" (T150-2016 Juzgado del Trabajo de Antofagasta) ${ }^{8}$.

En el caso de la libertad de expresión, particularmente en su vertiente de derecho a información, cabe señalar, que dicho derecho incluye "tanto la dimensión de derecho de crédito, que es el derecho a ser informado, como la vertiente de libertad publica, que es el derecho a informar e informarse".

De este modo, el trabajador tiene el derecho a hacer circular la información dentro de la empresa que le parezca pertinente, como a recibir de terceros la información que le parezca relevante ${ }^{10}$.

$8 \quad$ En el mismo sentido: "que al decidirse su desvinculación de la institución fundado en una mala calificación, que a su vez se determinó por la sanción administrativa impuesta al enjuiciarse negativamente las expresiones vertidas en un acto de defensa frente a una medida administrativa que lo afectaba, en opinión del tribunal constituye una vulneración del derecho a la libertad de opinión que tiene el funcionario denunciante, razón por la que se acogerá la acción en análisis" (T 540-2014 del 2 Juzgado del Trabajo de Santiago). De igual modo: "que será desestimada la alegación de la demandada consistente en que las declaraciones de la actora fueron difundidas con anterioridad a la fecha del despido y que por ello nadie ha censurado en forma previa sus declaraciones ni se le ha impedido hacerlo, puesto que si bien en la especie no se trata de un caso de censura previa, lo cierto es que, de acuerdo a lo expuesto en los acápites precedentes, el despido de la actora se vislumbra como un acto de represalia por haber ejercido en forma legítima su derecho a la libertad de expresión" (T 5-2012 del Juzgado del Trabajo de Puerto Montt).

9 MONEREO, J.L.: Los derechos de información de los representantes de los trabajadores. Civitas, Madrid, 1992. p 100.

10 ¿Tiene el trabajador derecho a recibir información del empleador? Como ya explicamos, la regla general es que los derechos fundamentales recogidos en la acción de tutela se caracterizan por una estructura negativa: suponen el deber de no intervenir dentro de 


\section{LÍMITES DE LA LIBERTAD DE EXPRESIÓN DEL TRABAJADOR}

La libertad de expresión del trabajador tiene tanto límites legales, como constitucionales.

En el primer caso, la norma que establece como causal de despido del trabajador -sin derecho a indemnización- "las injurias proferidas por el trabajador al empleador "(artículo 160, número 1, letra d.). El legislador ha mediado legalmente el cruce entre el derecho de la libre expresión del trabajador y la honra del empleador -sea persona natural o jurídica- ${ }^{-11}$.

Por tratarse de una restricción legal, debe ser leída de modo estricto y restringido: solo en casos especialmente "graves" -como lo exige la propia ley- debe entenderse justificado el despido del trabajador ${ }^{12}$.

Pero también existe un límite constitucional: el que derive de un eventual conflicto con otros derechos constitucionales de la empresa. Particularmente

las posiciones protegidas por el contenido del derecho, de ahí que su dimensión pasiva se exprese en prohibiciones para el sujeto que este afecto al derecho. Salvo que exista una exigencia normativa en ese sentido, que imponga ese deber positivo de entregar información. En el caso del derecho chileno, ese deber de información se establece para ciertos casos en el ámbito individual, como ocurre con el deber de informar sobre las normas del reglamento interno de la empresa (artículo 156 del Código del Trabajo), o en el ámbito del derecho colectivo, para la negociación colectiva y las relaciones sindicales en general (artículo 315 del Código del Trabajo). En este último caso, ver GONZALEZ, D.: Negociación Colectiva, Derecho de a la Información de los Sindicatos. Der ediciones, Santiago, 2017.

11 La honra tiene su fundamento inmediato en el atributo moral de la dignidad de la persona, lo que como señala Figueroa refiriéndose a la privacidad, impide que las personas jurídicas sean titulares de esta categoría de derechos fundamentales, atendido que se trataría de un "derecho personalísimo basado en el patrimonio moral del sujeto y en su dignidad, conceptos solo aplicables al ser humano y la personalidad humana". FIGUEROA, R.: Privacidad. Ediciones Universidad Diego Portales, Santiago, 2014. p 54.

12 Así, por lo demás, lo ha entendido la jurisprudencia judicial que ha sostenido en general que respecto de la libertad de expresión afirmar que, pese a que las declaraciones del trabajador son "imprudentes" no son constitutivas del incumplimiento grave de las obligaciones del contrato: "no es posible eludir que las expresiones proferidas públicamente son, a lo menos, imprudentes, es decir vertidas con desprecio o falta al cuidado debido, en tanto contienen una afirmación que aun falsa o verdadera (hecho desconocido para el Tribunal) coloca en situación de descrédito social a la empresa mandante y ello, en el análisis razonable de cualquier espectador externo, irroga temor a las represalias correlativas, en contrataciones comerciales futuras o incluso sobre la permanencia de la actual. Es decir, la actuación del trabajador, aun no siendo constitutiva de un grave incumplimiento al contrato de trabajo, importa una falta, descuido, o imprudencia que potencialmente afecta la posición comercial de su empleador" (T 1502016 del Juzgado del Trabajo de Antofagasta). 
con la denominada imagen empresarial, siempre que se entienda la misma como parte de un activo integrante del patrimonio empresarial, esto es, del derecho de propiedad. Lo anterior, porque la denominada "imagen corporativa" no puede formar parte del derecho a la honra, en cuanto, a nuestro juicio, ese derecho escapa de la órbita de las personas jurídicas al estar directamente fundado en la "dignidad individual" de la persona.

En cualquiera de estos casos de conflicto entre la libertad de expresión y otros derechos constitucionales de la empresa, debe tenerse en cuenta la especial relevancia del primero atendido la "naturaleza o índole" de la expresión y/o información. Si se trata de información de interés público debe ser entendido, entonces, como un derecho -prima facie- prioritario para ese caso concreto. Dicha "posición preferente" se sustenta en el fuerte nexo entre la libertad de expresión y la democracia. Como ha expresado la Corte Interamericana de Derechos Humanos "la libertad de expresión es indispensable para la formación de la opinión pública en una sociedad democrática" (Corte Interamericana de Derechos Humanos, caso Lagos del Campo vs, Perú, sentencia del 31 de agosto del 2017, párrafo 116).

En ese sentido, tendrían ese carácter de relevancia pública en ejercicio de la libertad de expresión por parte del trabajado, las siguientes hipótesis:

a.) la información o discurso de relevancia para la comunidad, específicamente por ser de "interés general", esto es, "todo hecho cuyo conocimiento puede tener interés para los ciudadanos, bien por las personas $o$ instituciones que intervienen en el mismo, bien porque objetivamente el asunto tiene trascendencia social, aunque sea meramente coyuntural"'13. En el caso de un trabajador ese discurso de interés general puede tratarse, por de pronto, de la difusión de propuestas políticas y sociales, pero también cuestiones referidas al ámbito empresarial en particular, como denuncias de evasión legal o fraude por parte del empleador, incumplimiento de normas sanitarias en los procesos productivos, etc.

La justificación de la especial relevancia de la libertad de expresión en este punto es que -como ha explicado la Corte Interamericana de Derecho Humanos "se trata de una piedra angular en la existencia misma de una sociedad democrática, Es indispensable para la formación de la opinión pública. Es también conditie sine qua non para que los partidos políticos, los sindicatos, las sociedades científicas y culturales, y en general, quienes deseen influir sobre la colectividad pueden desarrollarse plenamente.

13 PEREZ ROYO, J.: Curso de Derechos Constitucional. Marcial Pons, Madrid, 2002. p 443. 
Es, en fin, condición para que la comunidad, a la hora de ejercerse sus opciones, esté suficientemente informada" (Corte Interamericana de Derechos Humanos, sentencia "La Última tentación de Cristo", párrafo 68). Esta perspectiva preferente de la libertad de expresión en relación a la información de interés público o comunitario también es sostenida en la jurisprudencia europea: "La libertad de expresión constituye uno de los fundamentos esenciales de una sociedad de democrática, una de las condiciones primordiales de su progreso y del desarrollo de cada individuo. Sin perjuicio del apartado 2 del artículo 10, la libertad de expresión es válida no solamente para las informaciones o ideas acogidas favorablemente o consideradas inofensivas o indiferentes, sino también para aquellas que chocan, ofenden o inquietan: así lo quieren el pluralismo, la tolerancia y el espíritu de apertura sin los cuales no hay sociedad democrática" (Tribunal Europeo de Derechos Humanos, Asunto Lindon, Otchakovsky-Laurens y July con Francia, 22 de Octubre del 2007, párrafo 45).

En esa perspectiva, en un interesante caso de la jurisprudencia nacional se considera vulnerado el derecho a la libertad de expresión, por el despido de trabajador que ha realizado una denuncia que refleja una preocupación de la comunidad: "la acción del actor no puede ser calificada como una falta de probidad en los términos que la disposición legal mencionada establece, sino sencillamente su conducta reflejó el ejercicio del legítimo derecho consagrado en la Constitución Política de la Republica en el artículo 19 $\mathrm{N}^{\circ} 12$, referente al derecho que tiene todo ciudadano de este país a emitir opinión y el derecho a informar sin censura previa en cualquier forma y por cualquier medio que el Tribunal está obligado a resguardar. Así, el actor denunció una situación a petición de los dirigentes gremiales de Coquimbo, que consideraban injusta y que era necesario dar a conocer y revertirla, pero ello, en caso alguno, autoriza al empleador demandado a poner término a la relación laboral, como lo ha hecho, máxime cuando la situación acontecida es una situación que se refiere a todo el país, a todos los pescadores artesanales y a todos los industriales pesqueros, no es una situación que solo se refiera a esta cuarta región, si bien en esta zona tiene características especiales por el tema de las cinco millas marítimas" (T472014 Juzgado del Trabajo de la Serena).

b.) la información o discurso sobre el interés colectivo de los trabajadores. Se trata del discurso que represente cuestiones de interés que superan al trabajador-individuo que lo expresa, porque se conecta con el interés común de los trabajadores de una unidad empresarial, como por 
ejemplo denuncias sobre las condiciones de trabajo, o sobre el no pago de créditos laborales, cumplimiento de leyes laborales, etc.

La razón de esta prioridad parece obvia. La libertad de expresión puesta al servicio de los derechos de los trabajadores representa un interés que trasciende a los involucrados. Y ello porque el trabajo es una dimensión clave de la humanidad, donde la sociedad se juega parte relevante de los valores que la constituyen, como la dignidad personal, el reconocimiento, y la integración en la comunidad. De ahí, que la sociedad tenga interés en su conjunto de que las condiciones materiales y emocionales en que esa dimensión se desarrolla están a la altura de su relevancia para todos. Nexo este -el del trabajo y la comunidad- lucidamente retratado hace años por Estey: "el bienestar de los asalariados en las localidades industriales es la preocupación de todos. Como ciudadanos, empleadores, consumidores, trabajadores, de uno u otro modo todos estamos afectados por la prosperidad o la adversidad, la satisfacción o el malestar, la eficiencia o ineficiencia, la riqueza o la pobreza del asalariado. Estas verdades son particularmente aplicables a una democracia que por su naturaleza ha de admitir la igualdad del hombre, ha de afirmar el derecho igual a acceder a las cosas buenas de la vida y ha de tomar las medidas necesarias para la realización de estas oportunidades" 14 .

De ahí, por lo demás, el trato que de la jurisprudencia internacional recibe la libertad de expresión en estos casos: "Esta Corte reconoce que la emisión de información concerniente al ámbito laboral, por lo general, posee un interés público. En un primer término, deriva en un interés colectivo para los trabajadores correspondientes, y con un alcance especialmente determinado144, y más aún, cuando las opiniones trascienden al ámbito de un modelo de organización del Estado o sus instituciones en una sociedad democrática"(Corte Interamericana de Derechos Humanos, caso Lagos del Campo vs, Perú, sentencia del 31 de agosto del 2017, párrafo 111).

En ese caso pueden darse dos hipótesis: que dicho discurso sea sostenido por un trabajador cualquiera, en cuyo caso quedará al amparo constitucional de la libertad de expresión, o de un representante sindical de los trabajadores, donde el discurso quedará al amparo de la libertad sindical. En ambos casos, supone una protección reforzada como forma de garantizar que la expresión de ese trabajador sea el modo de dar voz al resto de los trabajadores.

14 ESTEY, J.A.: The Labor Problem. McGraw-Hill, New York, 1928. p 1. 
En estos casos, las restricciones de la libertad de expresión por parte de la empresa -que adopten la forma de despido u otra conducta empresarialdebe considerarse ilícita y lesiva del derecho fundamental respectivo. Salvo una justificación de peso extremo y prioritaria para la salvaguarda de otro interés constitucional en juego, la justificación empresarial debe ser considerada en estos casos insuficiente.

En cualquier caso, por último, cabe oponer como límite al ejercicio del derecho de la libertad de expresión -y en rigor, en ningún derecho fundamentales del trabajador- ni el denominando principio de buena fe, ni menos los arcaicos deberes éticos del trabajador. Eso, como parece obvio, vulnera el principio de primacía constitucional de los derechos fundamentales. Esto es especialmente relevante en el caso de la libertad de expresión, cuyo contenido -como explicamos antes- puede suponer la protección de discurso críticos, ácidos e hirientes para el empleador, poco compatibles con la idea de moderación que suele rodear la retórica jurídica de la moderna buena fe o de la anticuada lealtad ${ }^{15}$.

\section{BIBLIOGRAFÍA.}

BUDD, J.: El pensamiento sobre el trabajo. Tirant lo Blanch, Valencia, 2015.

FIGUEROA, R.: Privacidad. Ediciones Universidad Diego Portales, Santiago, 2014.

GONZALEZ, D.: Negociación Colectiva, Derecho de a la Información de los Sindicatos. Der ediciones, Santiago, 2017

GONZÁLEZ, F.: "Censura judicial y libertad de expresión: Sistema interamericano y derecho chileno". En: AAVV. Libertad de Expresión en Chile. Universidad Diego Portales, Santiago, 2006

LOVERA, D.: "Libertad de expresión, derecho de reunión y protesta en la Constitución". En: AAVV La Constitución chilena. Bassa et alt (editores). LOM editores, Santiago, 2015

15 Para una revisión crítica ver UGARTE, J. L:. "Los derechos fundamentales del trabajador y la buena fe en el debate español". En: Revista de Derecho, PUCV, $\mathrm{N}^{\circ} 36,2011$, Valparaíso. pp. 141-164. 
MARCIANI, B.: El derecho a la libertad de expresión y la tesis de los derechos preferentes. Palestra, Lima, 2004.

MEDINA, C.: "La libertad de expresión". En: AAVV Sistema Jurídico y Derechos Humanos, El derecho nacional y las obligaciones internacionales de Chile en materia de Derechos Humanos. Universidad Diego Portales, Santiago de Chile, 1996.

MONEREO, J.L.: Los derechos de información de los representantes de los trabajadores. Civitas, Madrid, 1992.

PEREZ ROYO, J.: Curso de Derechos Constitucional. Marcial Pons, Madrid, 2002. 